Erratum

\title{
Erratum to "Effectiveness of the Novel Herbal Medicine, KIOM-MA, and Its Bioconversion Product, KIOM-MA128, on the Treatment of Atopic Dermatitis"
}

\author{
Tae Ho Chung, ${ }^{1}$ Tae Jin Kang, ${ }^{2}$ Won-Kyung Cho, ${ }^{1}$ Ga Yeong Im, ${ }^{1}$ Geum Seon Lee, ${ }^{2}$ \\ Min Cheol Yang, ${ }^{1}$ Chang-Won Cho, ${ }^{3}$ and Jin Yeul $\mathrm{Ma}^{1}$ \\ ${ }^{1}$ Center for Herbal Medicine Improvement Research, Korea Institute of Oriental Medicine (KIOM), 483 Expo-ro, Yuseong-gu, \\ Daejeon 305-811, Republic of Korea \\ ${ }^{2}$ College of Pharmacy, Sahmyook University, 26-21 Kongnung 2-dong, Nowon-gu, Seoul 139-742, Republic of Korea \\ ${ }^{3}$ Regional Food Industry Research Group, Korea Food Research Institute, Sungnam 463-746, Republic of Korea \\ Correspondence should be addressed to Jin Yeul Ma; jyma@kiom.re.kr
}

Received 5 December 2013; Accepted 31 December 2013; Published 5 February 2014

Copyright (C) 2014 Tae Ho Chung et al. This is an open access article distributed under the Creative Commons Attribution License, which permits unrestricted use, distribution, and reproduction in any medium, provided the original work is properly cited.

In the whole paper, Lactobacillus acidophilus was incorrectly used. The correct name is Lactobacillus rhamnosus. The authors apologize for this error. 


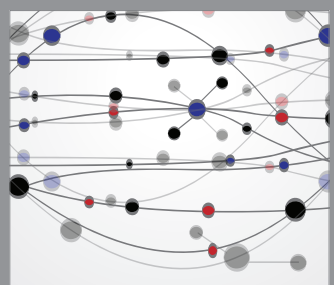

The Scientific World Journal
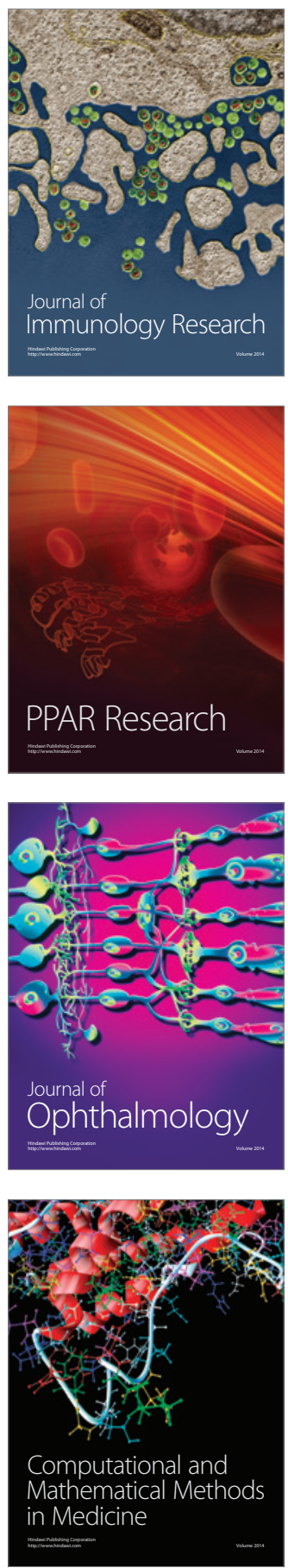

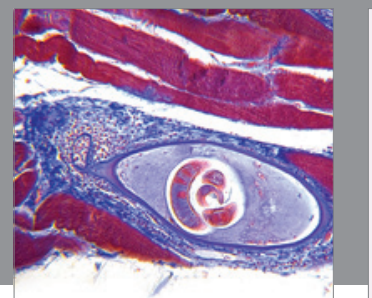

Gastroenterology

Research and Practice
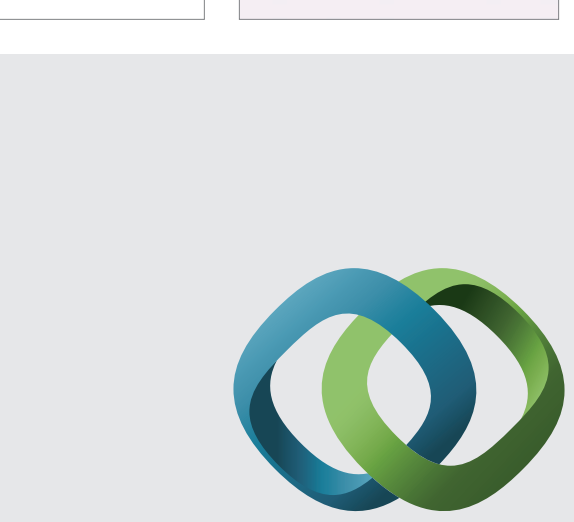

\section{Hindawi}

Submit your manuscripts at

http://www.hindawi.com
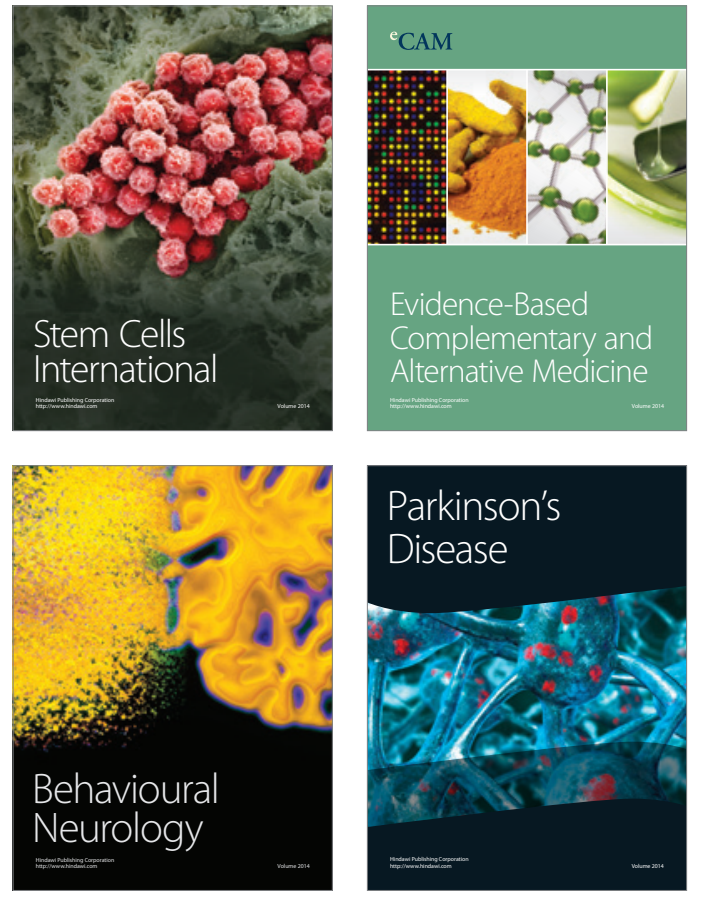
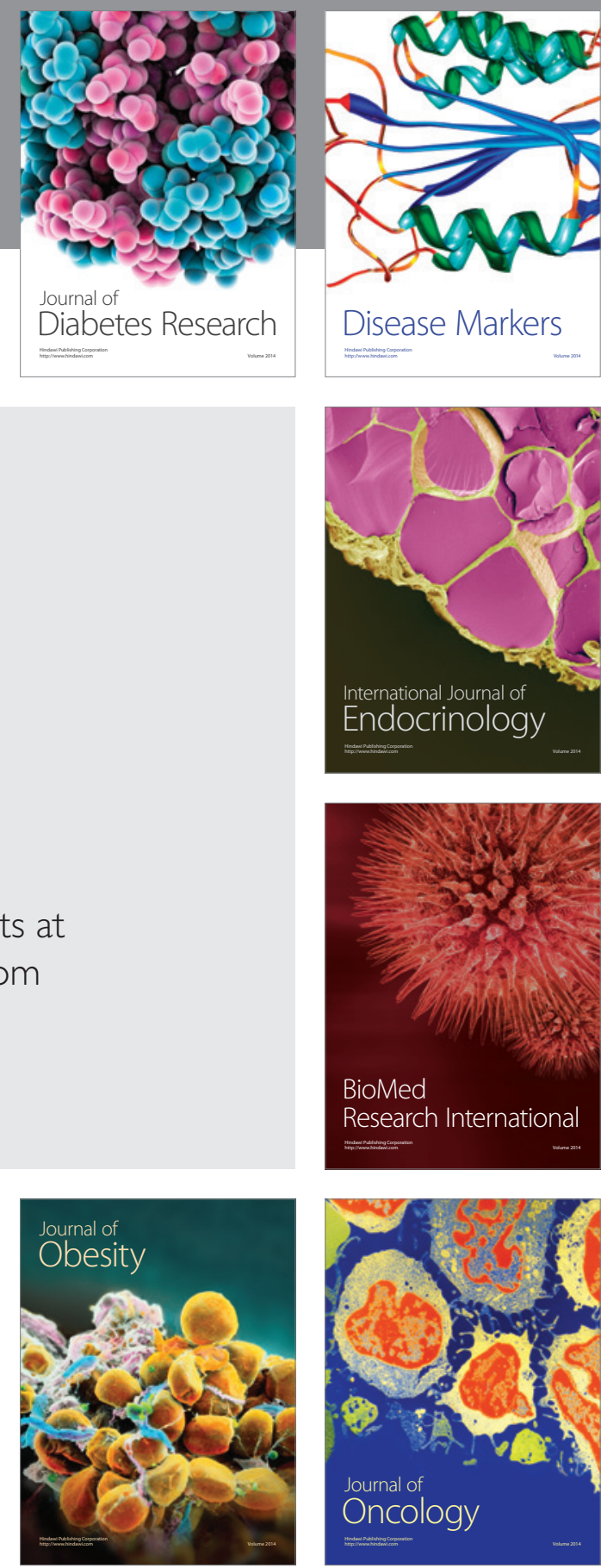

Disease Markers
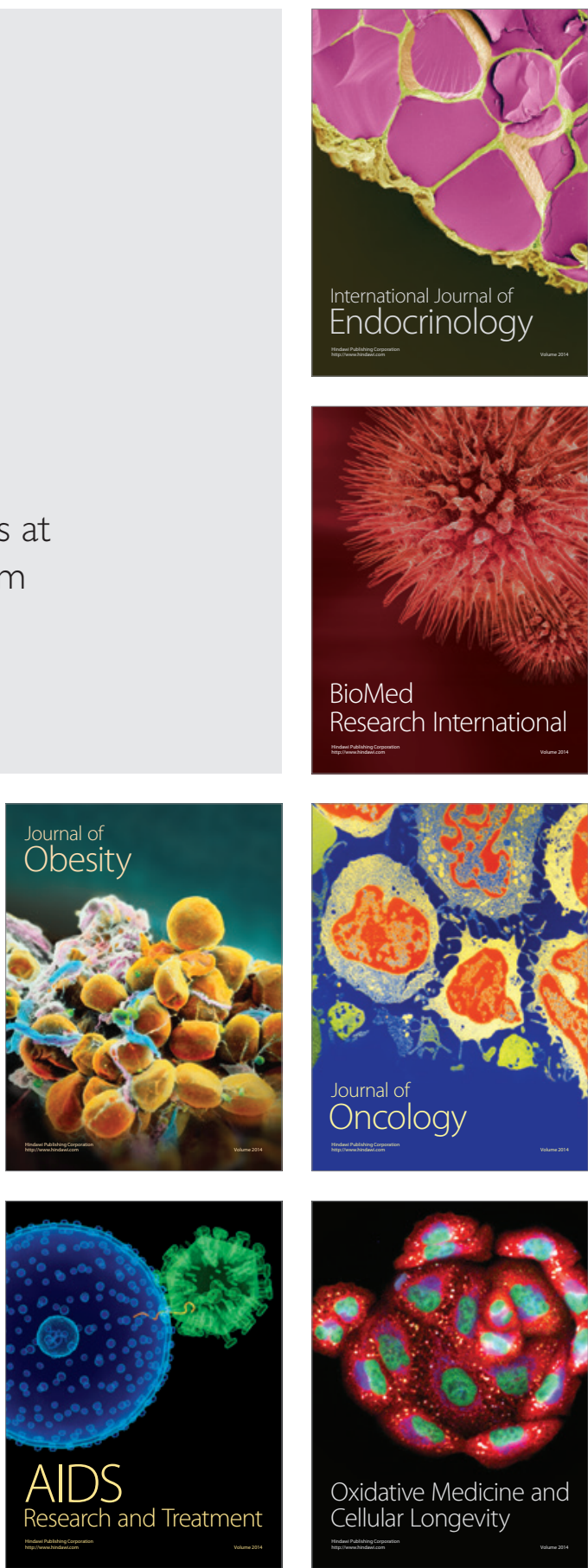